\title{
Modelo preditivo para diagnóstico da SEPSE em unidades de terapia intensiva.
}

\author{
Predictive model for SEPSIS in the intensive care \\ unit.
}

\section{Modelo predictivo para el diagnóstico de SEPSIS en unidades de cuidados intensivos.}

Lavoisier Morais de Medeiros

Ana Maria Gondim Valença ${ }^{2}$

Ulisses Umbelino dos Anjos ${ }^{3}$

RESUMO: O presente estudo teve como objetivo fornecer um modelo de decisão probabilístico para o diagnóstico precoce da sepse. Foram analisados os dados contidos nos prontuários de 100 indivíduos internados em uma UTI geral de um hospital público do interior do estado da Paraíba, no período de março a setembro de 2011. As variáveis estudadas foram: idade, sexo, diagnóstico inicial, temperatura axilar mínima e máxima, frequência cardíaca e respiratória, pressão parcial de oxigênio e de gás carbônico, nível sérico de lactato, potássio, sódio, contagem total de leucócitos, bastonetes e segmentados, dentre outras. Utilizou-se a regressão logística binária para determinação do modelo de predição, sendo os dados analisados pelo software SPSS versão 19.0. Constatou-se que 63\% dos participantes pertenciam ao sexo masculino, com idade média de 62,5 anos. Foram consideradas como variáveis explicativas: a temperatura axilar mínima, a temperatura axilar máxima, a pressão parcial de gás carbônico, o lactato, a contagem de leucócitos e o número de bastonetes. Através da curva ROC identificou-se o ponto de corte ideal para classificação dos indivíduos quanto à presença ou ausência da doença, o que contribuiu para confecção da regra de tomada de decisão para o diagnóstico precoce da sepse. Realizou-se a comparação do grau de concordância entre o resultado da hemocultura considerado como padrão-ouro para o diagnóstico da infecção e o modelo apresentado no estudo utilizando-se o coeficiente Kappa, sendo obtido um percentual de concordância de 0,93 , considerado como excelente. Demonstrou-se ser possível a detecção precoce 1 Mestre em Modelos de Decisão e Saúde pela Universidade Federal da Paraíba (UFPB). Professor Assistente da Universidade Federal de Campina Grande (UFCG). Professor do Instituto Federal da Paraíba - Campus Patos (IFPB) 2 Professora Titular do Departamento de Clínica e Odontologia Social da Universidade Federal da Paraíba (UFPB). Docente do Programa de Pós-graduação em Modelos de Decisão e Saúde da Universidade Federal da Paraíba (UFPB).

3 Professor Associado do Departamento de Estatística da Universidade Federal da Paraíba (UFPB). Docente do Programa de Pós-graduação em Modelos de Decisão e Saúde da Universidade Federal da Paraíba (UFPB). 
da sepse com a adoção de modelos estatísticos como o apresentado, entretanto, novos estudos com populações de diferentes UTIs devem ser realizados a fim de prover uma casuística melhor, tornando os resultados encontrados reproduzíveis em diferentes situações clínicas diárias.

Palavras-Chave: Unidade de Terapia Intensiva; Sepse; Modelo de Decisão.

ABSTRACT: The present study aimed to provide a probabilistic decision model for the early diagnosis of sepsis. It was analyzed the data contained in the records of 100 patients hospitalized in a general ICU of a public hospital in the state of Paraíba, in the period from March to September 2011. The variables analyzed were: age, gender, initial diagnosis of the participants, the minimum and maximum axillary temperature, heart rate and respiratory rate, partial pressure of oxygen and carbon dioxide, serum lactate, potassium, sodium, total leukocyte count, segmented rods and, among others. It was used binary logistic regression to determine the prediction model. Data analysis was performed using SPSS version 19.0. The findings showed that $63 \%$ of study participants were male, with a mean age of 62.5 years. Explanatory variables were considered: axillary temperature minimum, maximum axillary temperature, partial pressure of carbon dioxide, lactate, leukocyte count and the number of rods. ROC curve was possible to identify the optimal cutoff point to classify individuals according to the presence or absence of disease, which contributed to the making of the rule of decision for the early diagnosis of sepsis. Was performed to compare the degree of agreement between blood culture result considered the gold standard for diagnosis of infection and the model presented in this study using the Kappa coefficient, obtained a percentage of agreement of 0.93 is considered excellent. Despite the unprecedented nature of the research, proved to be early detection of sepsis with the adoption of statistical models as presented, however, further studies with different populations of ICUs should be performed in order to provide a better sample, making the results found reproducible in different clinical situations daily.

Keywords: Intensive Care Unit; Sepsis; Decision Model.

RESUMEN: Este estudio tuvo como objetivo proporcionar un modelo de decisión probabilística para el diagnóstico precoz de la sepsis. Los datos se analizaron en los registros médicos de 100 individuos ingresados en una UCI general de un hospital público en el estado de Paraiba, de marzo a septiembre de 2011. Las variables estudiadas fueron: edad, sexo, diagnóstico inicial, la temperatura mínima axilar y la frecuencia cardíaca máxima y la frecuencia respiratoria, la presión parcial de oxígeno y dióxido de carbono, el lactato sérico, potasio, sodio, el recuento total de leucocitos, varillas y específica, entre otros. Se utilizó la regresión logística binaria para determinar el modelo de predicción, y los datos analizados por el software SPSS versión 19.0. Se encontró que el 63\% de los participantes eran hombres, con una edad media de 62,5 años. Se consideraron como variables explicativas: la temperatura axilar mínimo, la temperatura máxima axilar, la presión parcial de dióxido de carbono, el lactato, el recuento de leucocitos y el número de varillas. A través de la curva ROC identificó el punto de corte óptimo para la clasificación de los individuos para determinar la presencia o ausencia de la enfermedad, lo que contribuyó a que la regla de toma de decisiones 
para el diagnóstico precoz de la sepsis. Se realizó para comparar el grado de concordancia entre el resultado del hemocultivo considerado como el patrón oro para el diagnóstico de la infección y el modelo en el estudio utilizando el coeficiente Kappa, y obtuvo un porcentaje de concordancia de 0,93 , considerado excelente. Demostrado que es posible la detección precoz de la sepsis con la adopción de modelos estadísticos tal como se presenta, sin embargo, otros estudios en poblaciones de diferentes UCI deben llevarse a cabo con el fin de proporcionar una mejor muestra, haciendo que los resultados reproducibles en diferentes situaciones clínicas diarias.

Palabras clave: Unidades de Cuidados Intensivos, Sepsis, Modelos de Decisión.

\section{INTRODUÇÃO}

As Unidades de Terapia Intensivas (UTI) são áreas de tratamento complexas destinadas à atenção aos indivíduos que necessitem de cuidados intensivos e que possam apresentar boa probabilidade de sobrevida. Seu surgimento ocorreu devido à necessidade de prestar uma assistência mais especializada e contínua a indivíduos com patologias graves ou de risco ${ }^{1}$.

A ideia de agrupar pacientes graves para proporcionar melhor assistência surgiu com Florence Nightingale, na Guerra da Criméia, em 1854. O aperfeiçoamento das intervenções terapêuticas e o desenvolvimento de unidades especiais para abrigar pacientes e recursos tecnológicos de alta complexidade transformaram as taxas de mortalidade por diversas patologias. Com a criação de áreas específicas e diferenciadas para a assistência intensiva nas últimas décadas, tornou-se viável a manutenção e a recuperação de pacientes com diversos tipos de doenças e quadros de instabilidade aguda delas decorrentes, hemodinâmica, ventilatória, metabólica, renal, entre outras²

O acompanhamento de pacientes com infecção é um desafio para a equipe multidisciplinar da UTI. Nos indivíduos admitidos com sintomas prévios à hospitalização, a infecção é considerada adquirida na comunidade, aqueles que desenvolvem infecção com mais de 48 horas após a admissão hospitalar, esta é considerada com intra-hospitalar ou nosocomial. As principais síndromes infecciosas que podem necessitar de admissão e terapia imediata na UTI são: a sepse, a pneumonia, a endocardite infecciosa, as infecções intraabdominais dentre outras, contudo a de maior prevalência e de pior prognóstico é a sepse ${ }^{3}$.

O termo sepse significa putrefação, decomposição da matéria orgânica por um agente agressor (bactérias, fungos, parasitas, vírus). Os termos infecção e sepse são geralmente utilizados de forma independente, no entanto a terminologia acaba simplificando uma relação complexa. O termo infecção está relacionado à presença de agente agressor em uma localização (tecido, cavidade ou fluido corporal) normalmente estéril, e o termo sepse está relacionado à consequente manifestação do hospedeiro, i.e., à reação inflamatória desencadeada frente uma infecção grave. A distinção entre os dois não é fácil, pois todo processo infeccioso desencadeia uma resposta do hospedeiro, e cada indivíduo apresenta um tipo de reação com magnitudes diferentes frente um determinado agravo ${ }^{4}$. 
A adoção de estratégias que possibilitem o rápido diagnóstico e tratamento da sepse é fundamental, uma vez que sua ocorrência está associada à significativa morbidade e mortalidade se tal quadro não for prontamente reconhecido e tratado ${ }^{5,6}$.

Diante dessa problemática surgem alguns questionamentos: Como avaliar a gravidade dos indivíduos internados na UTI de forma a garantir a melhor assistência? A conduta realizada está trazendo uma resposta satisfatória à saúde do paciente? Existe alguma forma de predizer a evolução de uma determinada afecção e assim reduzir seus malefícios ao cliente?

Vários escores prognósticos que associam diferentes parâmetros clínicos e laboratoriais como o Acute Physiology and Chronic Health Evaluation (APACHE II) e o Sepsis Related Organ Failure Assessment (SOFA) são utilizados para avaliar o risco de mortalidade em pacientes na UTI. Contudo, esses escores, apesar de apresentarem um alto poder preditivo positivo quanto à mortalidade, não são adaptados a realidade brasileira o que torna difícil a sua aplicação na prática clínica diária ${ }^{1}$.

Até 1988, esses índices prognósticos eram largamente utilizados nas Unidades de Terapia Intensiva brasileiras, sem, no entanto, serem validados quanto às diferenças morfofisiológicas existentes entre a população do Brasil e a dos Estados Unidos e da Europa, onde foram desenvolvidos ${ }^{1}$.

Com esse intuito, realizou-se um estudo multicêntrico aplicando o modelo APACHE II a 1734 pacientes internados em dez hospitais brasileiros e, posteriormente, os dados foram analisados pela equipe de William Knaus em Washington, demonstrando importantes diferenças de SMR (Standardized Mortality Rate) - relação entre a mortalidade prevista e a mortalidade observada. E de maneira geral, a mortalidade observada foi superior à estimada pelo modelo APACHE II $^{7}$.

No Brasil, foi desenvolvido um modelo de avaliação prognóstica individual para pacientes internados em Unidades de Tratamento Intensivo, o modelo denominado UNICAMP II. Este foi fruto da análise de um banco de dados oriundo de um hospital universitário brasileiro e comparado a outros modelos publicados na literatura como o APACHE II. Além de ter sido verificada sua validade e retratada a realidade brasileira, se pode salientar, entre seus méritos, o fato da estimativa de risco atribuir um escore único para todos os pacientes, independente da causa que motivou a internação na $\mathrm{UTI}^{8}$. Contudo, o modelo UNICAMP II avalia a mortalidade na unidade de tratamento intensivo, sem realizar uma correlação específica com a presença de Sepse.

Assim, no Brasil, estudos epidemiológicos que avaliem a mortalidade ocasionada pela sepse ainda são inexistentes. Dessa maneira, se torna imprescindível a implementação de um protocolo rigoroso de medidas, baseada em evidências científicas e norteadoras, voltado para a detecção precoce e predição da mortalidade, buscando, melhor otimizar a alocação dos recursos técnicos e financeiros e, assim, obtermos resultados mais eficientes no tratamento da sepse reduzindo a morbimortalidade e, por consequência, o seu impacto social. 
Diante do exposto, o presente estudo objetivou fornecer um modelo de decisão probabilístico para o diagnóstico precoce da sepse a partir de dados clínicos de pacientes internados em uma UTI geral de um hospital público do interior do estado da Paraíba.

\section{METODOLOGIA}

Trata-se de um estudo de campo, longitudinal com abordagem quantitativa, realizado na Unidade de Terapia Intensiva do Hospital Regional de Patos (HRP), durante o período de abril a outubro de 2011. A pesquisa foi aprovada pelo Comitê de Ética e Pesquisa das Faculdades Integradas de Patos (Parecer Número: 106/2011).

O HRP está situado na cidade de Patos, no Sertão da Paraíba, a $300 \mathrm{~km}$ da capital João Pessoa/ $\mathrm{PB}$, sendo um município de médio porte com população predominantemente urbana, totalizando 106.314 habitantes ${ }^{9}$. A Unidade de Terapia Intensiva do Hospital Regional de Patos (HRP) possui seis leitos, atendendo exclusivamente indivíduos adultos, caracterizando-se como UTI geral por receber pacientes acometidos por diversas patologias distintas, como indivíduos vítimas de doenças cardíacas, respiratórias, acidentes, distúrbios metabólicos, dentre outras.

Os dados foram coletados a partir dos prontuários de todos os indivíduos em internação na referida UTI no período de março a outubro de 2011. Este período coincidiu com a coleta de dados para elaboração de dissertação de Mestrado do Programa de Pós-graduação em Modelos de Decisão e Saúde da Universidade Federal da Paraíba (UFPB) perfazendo um total de 113. A amostra foi composta pelos prontuários de todos os pacientes que apresentaram todos os critérios de inclusão da pesquisa, num total de 100 indivíduos.

Fizeram parte do estudo aqueles indivíduos que obedeceram aos seguintes critérios de inclusão: possuir idade superior ou igual a 15 anos, estar internado na UTI do HRP durante o período de coleta dos dados, possuir em seus prontuários os registros de todas as variáveis de interesse no estudo, coletadas a partir de instrumento próprio confeccionado pelo pesquisador. Foram excluídos da pesquisa todos os indivíduos que não atenderam a esses critérios.

Para realização do estudo foi utilizado um questionário, elaborado pelo pesquisador que continha dados gerais do paciente, como idade e sexo, o diagnóstico inicial ao ser admitido na UTI e variáveis clínicas e bioquímicas como, temperatura axilar mínima (TAM) e máxima (TAMX) registrada nas 24 horas anteriores à coleta, frequência cardíaca (FC) e respiratória (FR), concentração sanguínea de gás carbônico $\left(\mathrm{PCO}_{2}\right)$ e de oxigênio $\left(\mathrm{PO}_{2}\right)$, níveis séricos de lactato (LAC), sódio, potássio, contagem de leucócitos totais (LT) e de bastonetes (Bast), resultado da hemocultura, número de dias de internação na UTI (Dias de UTI), uso de antibioticoterapia e alterações ocorridas no tratamento durante o período de internação. Todas as variáveis analisadas foram escolhidas com base na literatura pertinente à temática.

Os dados foram coletados exclusivamente pelo pesquisador, sempre pelo período da manhã, 
por volta das 8 horas. A adoção dessa medida se dá como forma de se reduzir a possibilidade de vieses de aferição com a padronização da coleta. Justifica-se também por ser no período matutino que ocorre a troca de profissionais plantonistas da unidade, tornando-se mais fácil o acesso das informações daqueles pacientes admitidos durante todo o dia anterior, inclusive no plantão noturno.

Para coleta das variáveis: idade, sexo, diagnóstico inicial ao ser admitido na UTI, número de dias de internação na UTI, uso de antibioticoterapia, dias de diagnóstico clínico de sepse e alterações ocorridas no tratamento durante o período de internação, temperatura axilar, frequência cardíaca e respiratória foram utilizados os registros contidos no prontuário do paciente. Para a mensuração da concentração sanguínea de gás carbônico $\left(\mathrm{PCO}_{2}\right)$ e de oxigênio $\left(\mathrm{PO}_{2}\right)$ foi realizada a gasometria arterial do paciente, utilizando o gasômetro modelo TI500 disponível na UTI. A contagem de leucócitos totais e de bastonetes foi realizada com base no leucograma e os resultados anotados no prontuário do paciente.

Após a coleta, os dados foram tabulados em planilha do programa Excel ${ }^{\circledR}$ for Windows, versão 2007, e analisados utilizando-se o software SPSS (Statistical Package of the Social Sciences), versão 19.0. Inicialmente, as variáveis foram categorizadas da seguinte maneira: zero "0" para ausência do fator esperado e um " 1 ” para a presença. Desta feita, após a categorização as mesmas ficaram distribuídas conforme o Quadro 1.

Para construção do modelo estatístico foi utilizada a regressão logística binária. Este modelo tem como objetivo descrever a relação entre a variável resposta y dicotômica, como por exemplo, o status de doença (a doença está presente ou ausente) e as variáveis preditoras $\mathrm{X}_{1}, \mathrm{X}_{2}, \ldots, \mathrm{X}_{\mathrm{J}}$. Nesse modelo a variável resposta poderá assumir apenas dois valores, 0 ou 1. Em geral, o valor 1 é usado para representar um "sucesso" ou o resultado que estamos mais interessados e 0 para representar o fracasso ${ }^{10}$.

Quadro 1 - Valores das variáveis no Modelo Logístico

\begin{tabular}{|c|c|c|}
\hline Variável Analisada & Valor "0" & Valor "1" \\
\hline TAM & $\mathrm{Se}>36^{\circ} \mathrm{C}$ & $\mathrm{Se}<36^{\circ} \mathrm{C}$ \\
\hline TAMX & $\mathrm{Se}<38^{\circ} \mathrm{C}$ & $\mathrm{Se}>38^{\circ} \mathrm{C}$ \\
\hline $\mathrm{FC}$ & $\mathrm{Se}<90 \mathrm{bpm}$ & $\mathrm{Se}>90$ que bpm \\
\hline FR & $\mathrm{Se}<20 \mathrm{irpm}$ & $\mathrm{Se}>20 \mathrm{irpm}$ \\
\hline LAC & $\mathrm{Se}<2 \mathrm{mmol} / \mathrm{L}$ & $\mathrm{Se}>2$ mol $/ \mathrm{mml}$ \\
\hline Sódio & Se entre $136-145 \mathrm{mmol} / \mathrm{L}$ & $\mathrm{Se}$ outro valor \\
\hline Potássio & Se entre $3,5-5,0 \mathrm{mmol} / \mathrm{L}$ & $\mathrm{Se}<3,5 \mathrm{mmol} / \mathrm{L}$ \\
\hline PCO & $\mathrm{Se}<45 \mathrm{mmHg}$ & $\mathrm{Se}>45 \mathrm{mmHg}$ \\
\hline PO 2 & $\mathrm{Se}$ entre $80-100 \mathrm{mmHg}$ & $\mathrm{Se}$ outro \\
\hline LT & $\mathrm{Se}<12.000$ & $\mathrm{Se}>12.000$ \\
\hline Bastonetes & $\mathrm{Se}<10 \%$ & $\mathrm{Se}>10 \%$ \\
\hline Segmentados & $\mathrm{Se}<7.000$ & $\mathrm{Se}>7.000$ \\
\hline Cultura & $\mathrm{Se}$ negativa & $\mathrm{Se}$ positiva \\
\hline Diagnóstico de Sepse & $\mathrm{Se}$ negativo & $\mathrm{Se}$ positivo \\
\hline
\end{tabular}

Tempus, actas de saúde colet, Brasília, 10(2), 143-165, jun, 2016. 
Foi considerada como variável resposta ou dependente o resultado da hemocultura por ser esse considerado o padrão-ouro para identificação da sepse e as demais variáveis como independentes. Para determinar quais variáveis independentes possuíam significado estatístico para determinação da presença ou não de sepse, realizou-se o teste Qui-quadrado $\left(\chi^{2}\right)$ para testar o grau de associação entre as variáveis, sendo considerado um nível de significância de 5\%, ou seja, p-valor $<0,05$.

Construiu-se a escala de risco relativo de cada uma das variáveis que apresentaram significado estatístico para o modelo logístico e a partir do cálculo do risco foi desenvolvido o "peso" que cada fator desempenhou no desfecho da doença. O peso atribuído foi utilizado posteriormente para determinação da variável ESCORE que indica a contribuição global das variáveis analisadas para surgimento do desfecho Sepse, sendo determinado numa escala numérica de 0 a 100 para melhor compreensão da sua importância clínica ${ }^{11}$.

Após a determinação dos valores da variável ESCORE, foi construída a Curva ROC para testar a relação entre esta variável e o padrão-ouro e, desta forma, avaliar a relação entre elas. A partir da definição das coordenadas da curva ROC procedeu-se o cálculo do ponto de corte ideal para caracterizar quais indivíduos apresentam probabilidade de desenvolver ou não a sepse. A determinação do Ponto de Corte da Curva ROC foi realizada a partir do índice de Youden ${ }^{12}$.

Por fim, avaliou-se o grau de concordância entre os diagnósticos realizados pelo modelo de decisão apresentado e o real dos dados analisados, utilizando do coeficiente Kappa.

\section{RESULTADOS}

Este estudo contou com a participação de 100 indivíduos, sendo $63 \%$ do sexo masculino e 37\% do feminino.

No Gráfico 1 está apresentada a distribuição dos participantes quando ao diagnóstico inicial (admissional) na UTI. Embora alguns indivíduos apresentassem mais de um diagnóstico admissional, para esta análise foi considerado apenas o diagnóstico principal, ou seja, aquele que justificou a necessidade de internação na unidade. Constata-se que os diagnósticos mais prevalentes foram: Pós-operatório imediato com 19\%, Infarto Agudo do Miocárdio (IAM) com 17\%, Acidente Vascular Encefálico Isquêmico (AVEI) com 13 \% e Insuficiência Renal Aguda (IRA) com 11\% dos casos analisados. 
Gráfico 1 - Diagnóstico inicial dos indivíduos internados na UTI. Patos-PB, 2011

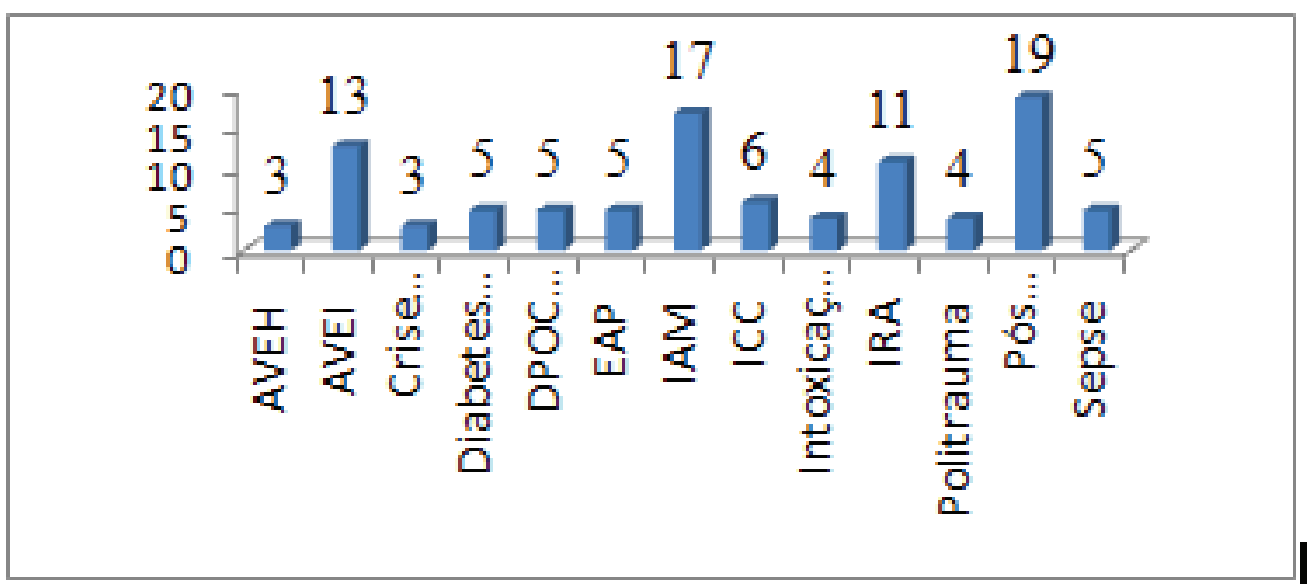

Os dados relativos à idade dos participantes da pesquisa encontram-se representados no Gráfico 2. A idade dos indivíduos variou entre 15 a 93 anos, com média de 62,5 anos e desvio padrão de \pm 9,23 . Nota-se também que as faixas etárias que apresentaram maior número de pacientes admitidos foram entre 61 a 70 anos com $31 \%$ e entre 51 a 60 anos com $27 \%$ do total.

Gráfico 2 - Distribuição dos participantes do estudo quanto à idade. Patos-PB, 2011.

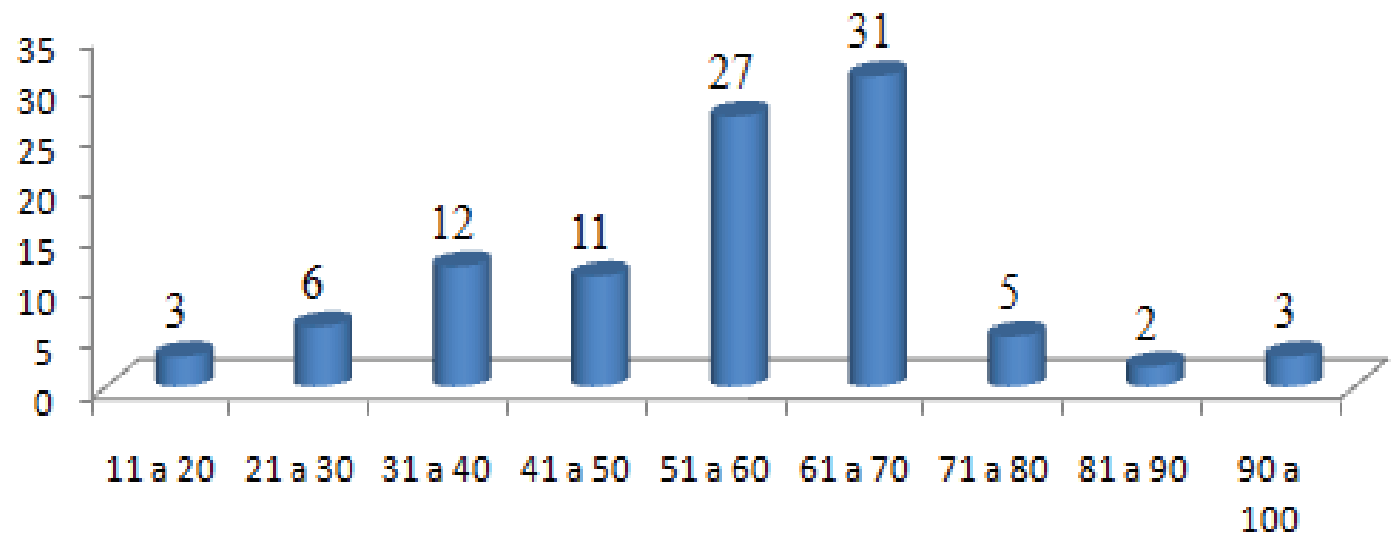

Com relação ao diagnóstico da sepse, dos 100 participantes da pesquisa 18 tiveram o diagnóstico clínico da síndrome o que nos fornece uma prevalência de $18 \%$. Contudo, entre esses pacientes diagnosticados, apenas 14 ou o equivalente a 77,8\% dos casos o resultado da hemocultura confirmou a presença de micro-organismo patogênico. Os agentes infecciosos encontrados na hemocultura foram Klebsiella pulmonare, Pseudomonas s.p. e Escherichia colli, com 9, 7 e 2 casos, respectivamente.

Outra variável analisada foi o número de dias de internamento dos indivíduos na UTI. Os dados demonstram que ocorreu uma variação entre 1 a 56 dias, com uma média de 6,8 dias e desvio padrão de $\pm 9,4$, permanecendo $73 \%$ dos participantes internados até 5 dias, $14 \%$ entre 6 a 10 dias e apenas $13 \%$ permaneceram mais de 10 dias na unidade. 
Os pacientes internados nas UTI devido ao seu quadro grave, geralmente são submetidos a diversas modalidades terapêuticas invasivas ou não, dentre elas, está a utilização de antibióticos. A utilização desta modalidade terapêutica desde o inicio do período de internação, foi observada na nossa pesquisa e pode-se constatar que $81 \%$ dos indivíduos internos na unidade fizeram uso de algum tipo de antibiótico e em 19\% não utilizaram.

Na prática clínica diária é comum o profissional de saúde reavaliar o tratamento proposto aos seus pacientes com objetivo de determinar se a resposta terapêutica está ou não adequada. Neste sentido, nosso estudo analisou a ocorrência de alterações no tratamento administrado aos indivíduos assistidos pela UTI. Verificou-se que em $88 \%$ dos indivíduos não ocorreu alteração na conduta terapêutica administrada e que em $12 \%$ essa mudança foi constatada.

Quanto ao procedimento estatístico desenvolvido para fornecer um modelo de decisão probabilístico para o diagnóstico precoce da sepse, este estudo se baseou na regressão logística binária, sendo incialmente aplicado o teste do Qui-quadrado para identificar associação entre as variáveis estudadas, adotando-se o nível de significância de 5\%.

Dentre os fatores analisados e apresentados na tabela 1 , os que apresentam o p- valor $<0,05$, e, portanto, são estatisticamente significantes, foram as variáveis: temperatura axilar mínima, temperatura axilar máxima, lactato, potássio, pressão parcial de gás carbônico, contagem de leucócitos totais, número de bastonetes e de segmentados. O p-valor da variável frequência respiratória foi de 0,052 , discretamente maior que o nível de significância determinado inicialmente, entretanto, devido a sua importância clínica no diagnóstico da patologia a mesma foi mantida na análise da regressão logística.

A variável tipo de micro-organismo, dias de internação na UTI e alterações no tratamento foram excluídas por não servirem para o diagnóstico clínico imediato (admissional), uma vez que seus valores só serão conhecidos a posteriori, a admissão do indivíduo na UTI, podendo sua manutenção no modelo logístico ser considerado com um fator de confundimento. Posteriormente o valor de concordância entre o diagnóstico clínico da sepse e o resultado da cultura foi avaliado através do coeficiente de Kappa e será demonstrado.

Desta feita, o Modelo de RL binário, teve como variável resposta o padrão-ouro para o diagnóstico da sepse que, de acordo com a literatura apresentada, é o resultado da hemocultura e como variáveis explicativas a temperatura axilar mínima, temperatura axilar máxima, a frequência respiratória, o lactato, nível sérico de potássio, a $\mathrm{PCO}_{2}$, o número de leucócitos totais, número de bastonetes e segmentados.

Contudo, após a inserção de todas as variáveis explicativas foi realizado o ajuste do modelo logístico, em que se retirava uma variável independente por vez e se observava a qualidade da resposta apresentada. Por fim, permaneceram como significativas as variáveis Temperatura Axilar mínima e máxima, Lactato, $\mathrm{PCO}_{2}$, Número de Leucócitos e Bastonetes, como pode ser visto na Tabela 1. 
Tabela 1 - P-valor das Variáveis do Estudo. Patos-PB, 2011.

\begin{tabular}{c|c}
\hline Variável & P-valor \\
\hline Temperatura Mínima & $<0,001$ \\
\hline Temperatura Máxima & 0,016 \\
\hline Frequência Cardíaca & 0,105 \\
\hline Frequência Respiratória & 0,052 \\
\hline Lactato & 0,014 \\
\hline Sódio & 0,282 \\
\hline Potássio & 0,042 \\
\hline PCO $_{2}$ & 0,005 \\
\hline PO $_{2}$ & 0,080 \\
\hline Leucócitos Totais & $<0,001$ \\
\hline Bastonetes & $<0,001$ \\
\hline Segmentados & $<0,001$ \\
\hline
\end{tabular}

A seguir, descreveremos a análise do modelo de regressão logística adotado. Pode-se observar na tabela 2 que o modelo testado apresentou um percentual de acerto a priori, ou seja, sem a adição das variáveis independentes de $82 \%$, o que indica que o modelo conseguiu determinar com precisão quando o indivíduo não apresentava sepse (verdadeiro negativo), porém, seu poder preditivo para o diagnóstico da presença da doença (verdadeiro positivo) é nulo.

Tabela 2 - Classificação do Modelo de Regressão a Priori. Patos-PB, 2011.

\begin{tabular}{|c|c|c|c|}
\hline \multirow{2}{*}{ Sepse } & \multicolumn{3}{|c|}{ Teste } \\
\cline { 2 - 3 } & Diagnóstico Sepse & \multirow{2}{*}{ Percentual de acerto } \\
\cline { 2 - 3 } & Negativo & Positivo & \\
\hline Diagnóstico negativo & 82 & 0 & $100 \%$ \\
\hline Diagnóstico positivo & 18 & 0 & $0 \%$ \\
\hline Percentual de acerto & 100 & 0 & $82 \%$ \\
\hline
\end{tabular}

Para avaliar a capacidade preditiva do modelo testado utilizou-se a estatística do Omnibus Tests do Modelo Logístico, que testou a hipótese de que todos os coeficientes da equação logística são nulos, o resultado do teste está descrito na tabela 3.

Tabela 3 - Omnibus Tests do Modelo Logístico. Patos-PB, 2011.

\begin{tabular}{|c|c|c|c|}
\hline & $\chi^{2}$ & df & Sig. \\
\hline Step & 87,007 & 6 & $<, 0001$ \\
\hline Block & 87,007 & 6 & $<, 0001$ \\
\hline Model & 87,007 & 6 & $<, 0001$ \\
\hline
\end{tabular}

Na avaliação sumária do modelo, demonstrada na tabela 4, temos a apresentação dos valores do Cox \& Snell R Square de 0,589 e o Nagelkerke R Square com valor de 0,952. Esses testes são considerados pseudos $\mathrm{R}_{2}$ e procuram indicar a proporção das variações ocorridas no log da razão de chance. Vê-se também o valor calculado para o Teste de Hosmer e Lemeshow que avalia o grau de acurácia do modelo logístico que nesse caso foi de 0,999. 
Tabela 4 - Avaliação Sumária do Modelo. Patos-PB, 2011.

\begin{tabular}{|r|r|r|r|rr|}
\hline Step & -2 Log likelihood & $\begin{array}{c}\text { Cox \& Snell R } \\
\text { Square }\end{array}$ & \multicolumn{1}{c|}{$\begin{array}{c}\text { Hosmer- } \\
\text { Lemeshow }\end{array}$} & \multicolumn{2}{|c|}{$\begin{array}{c}\text { Nagelkerke R } \\
\text { Square }\end{array}$} \\
\hline & 7,271 &, 589 & $\mathbf{9 9 9}$ & $\mathbf{9 5 2}$ \\
\hline
\end{tabular}

Com a inserção das variáveis independentes no modelo logístico, pode-se mensurar se ocorreu ou não alteração no seu poder preditivo. Na tabela 5 , observa-se que o percentual de acerto $a$ posteriori, ou seja, com a inserção das variáveis independentes chegou a 98\%. O mais importante a ser detectado é que anteriormente o modelo conseguia identificar com $100 \%$ de precisão os resultados verdadeiros negativos, porém, seu poder preditivo para o diagnóstico da presença da doença era nulo. Com a inserção das variáveis independentes a especificidade do modelo caiu para $98,8 \%$, contudo, a sua sensibilidade aumentou para $94,4 \%$.

Tabela 5 - Classificação do Modelo após inserção das variáveis independentes. Patos-PB, 2011.

\begin{tabular}{|c|c|c|c|}
\hline \multirow{2}{*}{ Observação } & \multicolumn{3}{|c|}{ Predição } \\
\cline { 2 - 3 } & \multicolumn{2}{|c|}{ Diagnóstico Sepse } & \multirow{2}{*}{ Percentual de acerto } \\
\cline { 2 - 3 } & Negativo & Positivo & \multirow{2}{*}{$98,8 \%$} \\
\hline Diagnóstico negativo & 81 & 1 & $94,4 \%$ \\
\hline Diagnóstico positivo & 1 & 17 & $\mathbf{9 8 \%}$ \\
\hline Percentual de acerto & 82 & 18 & \\
\hline
\end{tabular}

Para confecção do modelo logístico, é necessária a determinação dos valores dos coeficientes $\beta$ de cada variável, o que pode ser visto na tabela 6 .

Tabela 6- Valores de $\beta$ Para as Variáveis do Modelo Logístico. Patos-PB, 2011.

\begin{tabular}{|c|c|c|}
\hline Variável & Valor de $\boldsymbol{\beta}$ & p-valor \\
\hline Temperatura mínima & 35,845 & $<0,001$ \\
\hline Temperatura máxima & 18,738 & 0,010 \\
\hline Lactato & 18,408 & $<0,001$ \\
\hline $\mathrm{PCO}_{2}$ & 0,467 & 0,003 \\
\hline Leucócitos totais & 16,488 & $<0,001$ \\
\hline Bastonetes & 56,592 & $<0,001$ \\
\hline Constante & $-54,193$ & $<0,001$ \\
\hline
\end{tabular}

A partir dos valores apresentados acima a equação logística do modelo testado ficou determinada por:

Para determinação da curva ROC, foi realizada a comparação entre o padrão-ouro e variável ESCORE como descrito anteriormente. O gráfico apresentou área sob a curva de 0,946 (Figura 1). 
Figura 1 - Curva ROC entre o padrão-ouro e o Escore. Patos-PB, 2011

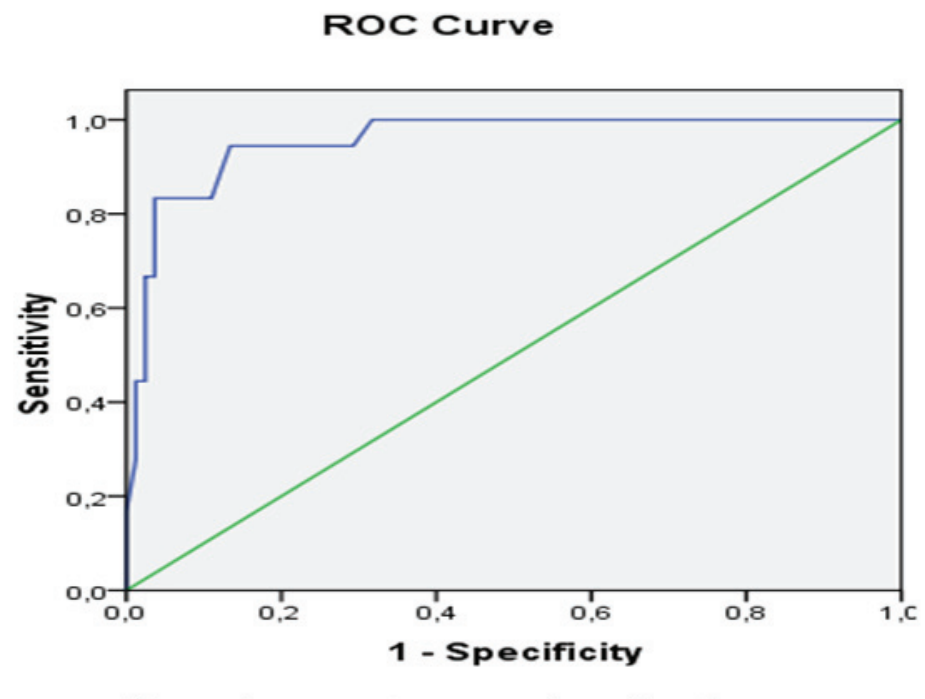

Diagonal segments are produced by ties.

Com base nos dados das coordenadas da curva ROC apresentados na tabela 7 e utilizando-se o Índice de Youden também descrito anteriormente foi possível determinar o ponto de corte ideal para o diagnóstico da presença ou não de sepse. Portanto, ficou determinado como ponto de corte ideal o valor 24 .

Tabela 7 - Coordenadas da Curva ROC

\begin{tabular}{|c|c|c|}
\hline Positivo $\mathbf{s e} \geq 1$ & Sensibilidade & 1 - Especificidade \\
\hline-1 & 1 & 0,598 \\
\hline 3,5 & 1 & 0,463 \\
\hline 8 & 1 & 0,378 \\
\hline 10 & 1 & 0,366 \\
\hline 13,5 & 1 & 0,354 \\
\hline 18,5 & 1 & $\mathbf{0 , 1 9 5}$ \\
\hline $\mathbf{2 4}$ & $\mathbf{1}$ & 0,183 \\
\hline 27,5 & 0,889 & 0,134 \\
\hline 29 & 0,889 & 0,122 \\
\hline 31 & 0,889 & 0,098 \\
\hline 33 & 0,889 & 0,098 \\
\hline 38,5 & 0,833 & 0,098 \\
\hline 44 & 0,778 & 0,085 \\
\hline 49,5 & 0,722 & 0,073 \\
\hline 55 & 0,722 & 0,061 \\
\hline 58 & 0,722 & 0,061 \\
\hline 62,5 & 0,667 & 0,061 \\
\hline 65,5 & 0,556 & 0,037 \\
\hline 69 & 0,5 & 0,037 \\
\hline 72,5 & 0,444 & \\
\hline & & Continua \\
\hline
\end{tabular}


Continuação

\begin{tabular}{|c|c|c|}
\hline 74 & 0,389 & 0,037 \\
\hline 76 & 0,389 & 0 \\
\hline 78 & 0,333 & 0 \\
\hline 80 & 0,278 & 0 \\
\hline 84,5 & 0,222 & 0 \\
\hline 90,5 & 0,056 & 0 \\
\hline
\end{tabular}

Com o conhecimento do ponto de corte ideal foi possível a elaboração da regra de decisão para o diagnóstico da sepse segundo o modelo estudado, que ficou assim determinada, como demonstrado na figura 2.

Figura 2 - Fluxograma de decisão para o diagnóstico da Sepse em UTI. Patos-PB, 2011

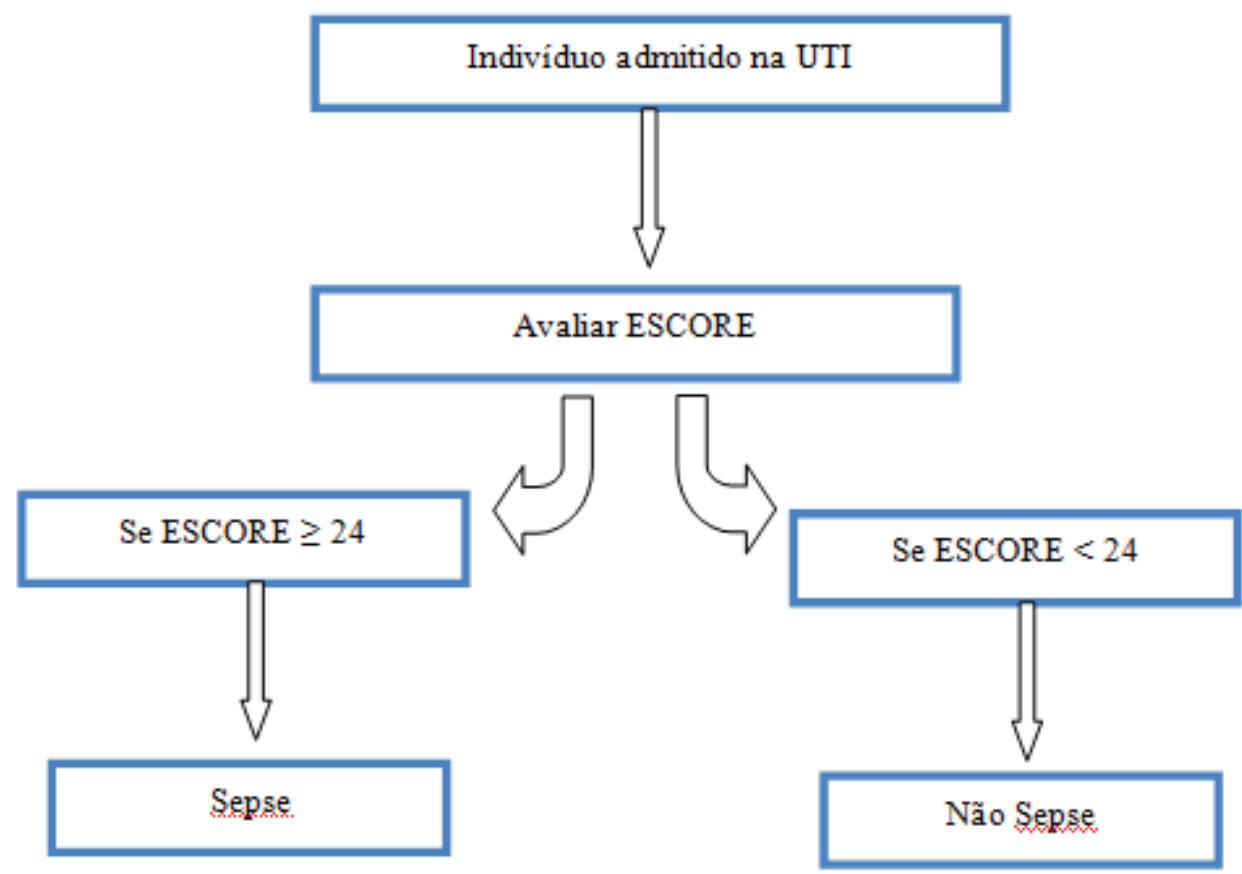

Elaborado pelos autores

Uma vez determinados o modelo logístico, o ponto de corte ideal para a classificação dos indivíduos e a regra de decisão que define os indivíduos como portadores ou não de sepse fica a pergunta: será que essas informações são realmente confiáveis para o diagnóstico da síndrome? Ou estamos diante de mais um modelo estatístico teórico com pouca ou nenhuma aplicação na prática clínica de fato?

Para solucionar esse questionamento comparamos o desempenho do modelo aqui proposto com o existente. Contudo, ressalta-se que não existe um modelo próprio para previsão do diagnóstico da sepse e, na prática clínica, o diagnóstico da sepse é estabelecido a partir de dados clínicos e a realização da hemocultura, que é considerada o padrão- ouro para confirmar ou excluir o diagnóstico, muito embora não apresente uma sensibilidade maior que 85\%. 
Todavia, mesmo sendo a hemocultura o padrão-ouro, existem muitos resultados falso-negativos o que torna a decisão clínica de tratamento ainda mais complexa uma vez que a não intervenção no quadro séptico poderá levar a morte do paciente. Diante do exposto, o modelo aqui apresentado poderá ser utilizado para subsidiar a tomada de decisão em pacientes com quadro clínico e laboratorial de sepse aumentando a confiabilidade do diagnóstico e a possibilidade de recuperação do indivíduo.

Diante da situação resolvemos realizar a comparação do desempenho do modelo aqui proposto com a hemocultura utilizando o cálculo da Sensibilidade, Especificidade, do Valor Preditivo Positivo e Negativo, da Razão de Verossimilhança Positiva e Negativa e do coeficiente Kappa.

Podemos observar na tabela 8, que se obteve uma Sensibilidade de 98,8\%, uma Especificidade de 94,4\%, o Valor Preditivo Positivo foi de 98,8\% e o Negativo de 94,4\%, a Razão de Verossimilhança Positiva 17,8 e a Negativa de 0,0; já o Coeficiente Kappa para comparação do percentual de concordância entre o padrão-ouro e o modelo apresentado foi 0,93 ; enquanto esses valores quando comparados o Padrão-ouro com o diagnóstico clínico da sepse foram de 95,1\%; 77,8\%; 95,1\%; $77,8 \% ; 4,3 ; 0,1$ e 0,73 respectivamente, demonstrando que o modelo apresentado é confiável.

Tabela 8 - Comparação da confiabilidade do Modelo testado, Patos-PB, 2011

\begin{tabular}{|c|c|c|}
\cline { 2 - 3 } & Modelo Testado/ Padrão-Ouro & $\begin{array}{c}\text { Diagnóstico Clínico/ Padrão- } \\
\text { Ouro }\end{array}$ \\
\hline Sensibilidade & 98,8 & 95,1 \\
\hline Especificidade & 94,4 & 77,8 \\
\hline Valor Preditivo Positivo & 98,8 & 95,1 \\
\hline Valor Preditivo Negativo & 94,4 & 77,8 \\
\hline Razão de Verossimilhança Positiva & 17,8 & 4,3 \\
\hline Razão de Verossimilhança Negativa & 0,0 & 0,1 \\
\hline Coeficiente Kappa & 0,93 & 0,73 \\
\hline
\end{tabular}

\section{DISCUSSÃO}

Entre os 100 indivíduos pesquisados constatou-se um predomínio do sexo masculino com 63\% do total. Esses achados são condizentes com outros estudos realizados em unidades de terapia intensiva no Brasil que encontraram, respectivamente, um percentual de 54,9\% e 58,2\% de internos do sexo masculino ${ }^{12,13}$. Com relação ao diagnóstico inicial, pode-se observar que os participantes do estudo possuíam diagnóstico de diferentes áreas da clínica e cirurgia o que se deve ao aspecto generalista da UTI analisada. Ao ser avaliada uma unidade de cuidados intensivos geral foi observado que as causas mais frequentes de internação eram hipertensão arterial sistêmica com $15 \%$ dos diagnósticos, infarto agudo do miocárdio com 12,5\%, insuficiência renal aguda e crônica com $10 \%$ e acidente vascular encefálico com $7,5 \%{ }^{14}$.

No presente trabalho, ao ser analisada a idade dos membros da amostra, observou-se que $41 \%$ dos indivíduos tinham mais de 60 anos, corroborando com estudo realizado na UTI do Hospital 
Regional da Asa Norte (HRAN), no Distrito Federal, na qual foi constatado que a maior parte dos pacientes pertencia à categoria acima de 60 anos $(29,9 \%)^{14}$. Este fato pode ser explicado em razão de, no Brasil, assim como nos países desenvolvidos, estar ocorrendo o fenômeno conhecido como transição demográfica, resultante da diminuição na taxa de natalidade e do aumento da longevidade da população. Como consequência, é cada vez maior o número de pacientes idosos internados nas Unidades de Terapia Intensiva ${ }^{16}$.

A incidência de sepse na unidade pesquisada foi de $18 \%$. Contudo, esse valor que a princípio parece ser muito elevado encontra-se dentro dos parâmetros de incidência da síndrome relatados mundialmente. As taxas de sepse relatadas na literatura podem variar de acordo com as características de cada local e a complexidade dos procedimentos realizados, podendo haver situações nas quais sua incidência chegue de 10 a 16\%, como em alguns países da Europa e nos Estados Unidos ou mesmo níveis extremante altos como em torno de 27 a $33 \%$ em nações pouco desenvolvidas ${ }^{17}$. A incidência média anual é de 51,5 casos por 100.000 habitantes adultos

Após a realização da hemocultura, três diferentes micro-organismos foram identificados nos sujeitos do estudo: Klebsiella Pneumoniae, Pseudômonas spp e Escherichia coli. Os principais agentes causadores da sepse em pacientes de UTI são Escherichia coli e Pseudomonas aeruginosa com 18,55 e 15,5\% do total de indivíduos infectados ${ }^{18,19}$. A avaliação de hemoculturas realizadas em pacientes da UTI de um hospital escola de Goiânia/GO demostrou que, dentre as bactérias Gram negativas, Pseudomonas spp e Klebsiella pneumoniae foram frequentemente registradas, corroborando os resultados do nosso estudo ${ }^{18}$. Estudo desenvolvido na China constatou que dentre as espécies mais prevalentes estavam Pseudomonas aeruginosa, Escherichia coli e Klebsiella pneumoniae ${ }^{21}$.

No ambiente hospitalar, principalmente nas UTI onde os pacientes são submetidos a inúmeros procedimentos invasivos quanto maior for a permanência neste setor, maior a probabilidade do desenvolvimento de processos infecciosos decorrentes de agentes microbiológicos oportunistas.

Um período de internação maior que 72 horas em UTI está associado a um risco 1,88 maior de óbito decorrente de infecções graves ${ }^{22}$. Entretanto, em nossa pesquisa não foi revelada relação direta entre o tempo de internação e o surgimento da sepse, fato esse que pode ser explicado pelo intenso controle de normas e rotinas da unidade estudada o que contribui para diminuição do risco de contaminações.

Um dos grandes problemas enfrentados atualmente pela medicina é a multirresistência microbiana que acaba levando ao uso de drogas cada vez mais potentes e onerosas para os serviços de saúde, sobretudo os serviços públicos. O uso racional de antibióticos não apenas diminui custos, mas, principalmente preserva a vida dos indivíduos infectados. Seu uso indiscriminado levou ao surgimento de cepas resistentes que ceifam vidas diariamente muito afora. 
Estudos apontaram que a administração não criteriosa de antibióticos aos pacientes internos em UTI neonatal ${ }^{23}$ e de idosos ${ }^{24}$ se constitui em um dos principais fatores relacionados com o surgimento de sepse devido a organismos multirresistentes. A ocorrência de modificações no tratamento desses pacientes sem a adoção de medidas criteriosas, como o antibiograma, aumenta em até 2,23 vezes a chance do desenvolvimento da sepse.

Os dados deste estudo demonstraram que $81 \%$ dos participantes fizeram uso de antibióticos durante o período de internação, tendo em muitos casos o início da administração dos mesmos ocorrido desde o primeiro dia de internação na unidade, sem, portanto o conhecimento do resultado hemocultura e, consequentemente, do antibiograma. Esse fato poderá ter contribuído para um aumento da incidência de casos diagnosticados de sepse por levar ao desencadeamento de resistência microbiana.

A possibilidade de prever com exatidão os prognósticos, como a mortalidade dos pacientes, ou a qualidade de vida futura de um indivíduo é especialmente importante porque ela pode servir para orientar as decisões individuais ou de grupo. A compreensão dos elementos importantes que entram no desenvolvimento de um modelo de previsão prognóstica pode ajudar aos profissionais de saúde a utilizarem esses instrumentos na prática cotidiana. Na medida em que os métodos para prever os prognósticos individuais sejam validados e mais amplamente aceitos. O conhecimento da estatística e da medicina baseada em evidência trouxe o suporte necessário para uma nova era de índices matematicamente comprovados como eficazes.

No presente estudo procurou-se analisar a influência de diversas variáveis clínicas para definição do diagnóstico precoce da sepse. Os dados obtidos demonstram que as variáveis que apresentaram valor estatístico para determinação do modelo preditivo para a doença foram a temperatura axilar mínima e máxima, a pressão parcial de gás carbônico, a concentração sérica de lactato, a contagem de leucócitos e o número de bastonetes. Contudo, podemos nos perguntar como interpretar clinicamente essas informações?

O processo de controle da temperatura corporal é bastante complexo mediado principalmente pelo hipotálamo através das áreas de produção, conservação e dissipação de calor. A temperatura se mantém estável graças ao equilíbrio entre a produção e a perda de calor pelo corpo. A produção de calor ocorre pela realização de reações químicas necessárias ao processo digestivo e pela contração dos músculos esqueléticos. A perda de calor se dá quando a temperatura ambiente está abaixo da temperatura corpórea - por irradiação, quando os objetos de diferentes temperaturas não estão em contato ou por condução quando a base de troca de calor é feita por contato direto ${ }^{25}$.

No paciente com quadro séptico esse mecanismo de regulação corporal encontra-se alterado uma vez que a exposição ao agente infeccioso e suas repercussões sistêmicas irão levar ao comprometimento progressivo da resposta do hipotálamo às mudanças de temperatura do meio interno do indivíduo, podendo ocorrer a elevação ou diminuição extrema da temperatura da pele do 
paciente. Como descrito anteriormente, para o diagnóstico da SIRS a temperatura do cliente deve apresentar-se em duas faixas distintas: menor que $36^{\circ} \mathrm{C}$ ou maior que $38^{\circ} \mathrm{C}$.

De acordo com os dados pesquisados aqueles indivíduos que apresentaram variações da temperatura dentro das faixas citadas acima apresentaram risco significativo para o surgimento da sepse, mas, por que isso ocorreu? A resposta para essa questão é bastante complexa, porém pode ser explicada por duas razões distintas: Primeiro, em uma UTI os sinais vitais são avaliados periodicamente, no caso da unidade avaliada a cada 2 horas durante todo o plantão de 24 horas. Desta feita, elevações discretas da temperatura axilar como o registro de temperatura na ordem de 35,9 ou $38,1^{\circ} \mathrm{C}$, por exemplo, são registradas e notificadas automaticamente a equipe médica que providencia imediatamente a administração de procedimentos terapêuticos para o controle dessas alterações.

Segundo, durante o desenvolvimento do processo infeccioso o organismo irá alterar o metabolismo basal como forma de combater de maneira mais eficiente a injúria sofrida, com isso as reações químicas próprias do processo metabólico puderam ser retardadas ou aceleradas de acordo com as necessidades energéticas do indivíduo o que levará, consequentemente, ao incremento ou decrescimento da temperatura interna e, por conseguinte a da região axilar.

O controle da oferta de sangue pelo organismo ocorre de forma autonômica utilizando a bomba cardíaca. Entretanto, em situações de sobrecarga sistêmica como no paciente acometido por Sepse, a quantidade da oferta de sangue para os tecidos periféricos encontra-se drasticamente diminuída. São comuns distúrbios de hipoperfusão capilar decorrentes principalmente da hipotensão gerada pela resistência vascular periférica, como consequência há um aumento do trabalho da bomba cardíaca o que ocasiona reflexamente elevação da freqüência de batimento do coração ${ }^{26}$.

O processo respiratório é controlado de duas maneiras distintas: de forma central pelo centro respiratório localizado na porção dorsal e ventral do bulbo e de maneira periférica pelos receptores carotídeos e aórticos. $\mathrm{O}$ controle central ocorre principalmente devido a ação dos íons $\mathrm{H}+$ nestes centros o que ocasiona a elevação da frequência respiratória e, consequentemente, redução do desequilíbrio ácido/base existente. Já a regulação periférica ocorre pela avaliação da concentração sanguínea de oxigênio, na qual discretos decréscimos nessa concentração levam a elevação da frequência respiratória como mecanismo compensatório.

No indivíduo com sepse teremos um aumento do metabolismo geral, o que elevará a demanda de oxigênio para que as reações químicas ocorram, ademais, devido ao desequilíbrio metabólico instalado, rapidamente se instalará uma acidose metabólica que se não corrigida levará o paciente a morte. Um dos mecanismos que o corpo irá utilizar para tentar frear esse processo será o aumento da FR como forma de corrigir as alterações metabólicas existentes, pelo incremento da oferta de oxigênio e eliminação do $\mathrm{PCO}_{2}$ presente nos gases respiratórios ${ }^{27}$. 
Diante do exposto, como consequência das alterações metabólicas decorrentes do processo séptico, o organismo irá sofrer alterações da concentração sérica de lactato e da concentração sanguínea de gás carbônico, fatos esses registrados em nossa pesquisa. Outrossim, até o presente momento não há consenso na literatura sobre o significado clínico dos componentes individuais da acidose metabólica, com exceção do lactato para o desenvolvimento da sepse, fato que acredita-se ter conseguido explicar ${ }^{27,28}$. Neste contexto, salienta-se que os efeitos hemodinâmicos deletérios da acidose láctica grave são amplamente sugeridos pelos dados experimentais, embora não sejam plenamente confirmados por estudos em humanos ${ }^{29}$.

Os leucócitos são as células de defesa do nosso organismo e são muito menos numerosos que os eritrócitos no sangue circulante. Eles têm origem na medula óssea e dividem-se em três classes: os granulados que constituem de $50 \%$ a $60 \%$ de todos os leucócitos e se apresentam na forma de neutrófilos, eosinófilos e basófilos (bastonetes); os linfócitos e os monócitos. Os bastonetes ocorrem no sangue normal em número muito pequeno, aproximadamente $0,5 \%$ do total dos leucócitos, e têm como função a reparação tecidual em processos alérgicos ou inflamatórios agudos ${ }^{25}$.

Na ocorrência de estados infecciosos como o existente durante a sepse o organismo é estimulado a elevar a produção dos leucócitos na tentativa de limitar a extensão da lesão tecidual, levando ao desenvolvimento da leucocitose. Diante do agravamento do quadro infeccioso um processo inflamatório refratário é desencadeado, visando restaurar a integridade local o que ocasionará o aumento da quantidade de bastonetes podendo seu percentual se elevar para $10 \%$ ou mais do total das células brancas presentes no sangue.

Para avaliar a confiabilidade do modelo logístico apresentado, utilizamos diversos testes estatísticos próprios para a regressão logística como o Omnibus Tests, o Cox \& Snell R Square, Nagelkerke R Square e o teste de Hosmer e Lemeshow.

O Omnibus test calculado foi $<0,0001$, com esse resultado rejeitamos a hipótese nula, ou seja, podemos afirmar que a introdução das variáveis independentes contribuem para melhorar a qualidade preditiva do modelo. Isso pode ser constatado quando comparamos o valor preditivo do modelo a priori e a posteriori, no qual o seu percentual de acerto alterou-se de 82 para $98 \%$.

O Cox \& Snell R Square e o Nagelkerke R Square são considerados pseudo R Square, sendo comparado ao R-Quadrado da Regressão Linear. Os valores de 0,589 e 0,952 respectivamente, indicam que 58,9 \% das variações ocorridas no $\log$ da razão de chances são explicadas pelo conjunto das variáveis independentes (TAM, $\mathrm{PCO}_{2}$, dentre outras) e que o modelo apresentado é capaz de explicar cerca de $95,2 \%$ das variações registradas na variável dependente, o que o torna bastante confiável ${ }^{30}$.

Para concluirmos a avaliação da confiabilidade do modelo de regressão logística apresentado, devemos observar o Teste de Hosmer e Lemeshow. Este teste tem o objetivo de testar a hipótese de 
que não há diferença significativa entre os resultados preditos pelo modelo e os observados. $\mathrm{O}$ valor calculado de 0,999 nos leva a aceitar a hipótese de nulidade, o que significa na prática que nosso modelo pode ser utilizado para estimar a probabilidade de um determinado indivíduo desenvolver sepse em função das variáveis independentes.

A área sob a curva ROC é uma medida do resumo usual do desempenho de um teste, uma vez que ela é estimada levando-se em consideração todos os valores da sensibilidade e da especificidade. Um teste totalmente incapaz de discriminar indivíduos doentes e não doentes teria uma área sob a curva de 0,5. Quanto maior a capacidade do teste em discriminar os indivíduos segundo dois grupos, mais a curva se aproxima do canto superior do gráfico, e a área sob a curva será próxima a 1 . No presente estudo a área sob a curva foi de 0,946 , o que representa uma alta capacidade de discriminação entre os indivíduos que desenvolveram a sepse e aqueles que não a apresentaram ${ }^{31}$.

Ao se observar as informações referentes à confiabilidade do modelo apresentado através da avaliação da Sensibilidade, Especificidade, VPP, VPN, da Razão de Verossimilhança Positiva $(\mathrm{RV}+)$ e Negativa (RV-) e do valor do Coeficiente Kappa, pode-se constatar que o modelo testado demonstrou ter um excelente desempenho geral quando comparado com o padrão-ouro, podendo ser ressaltado principalmente o valor da $\mathrm{RV}+$ de 17,8 , o que implica dizer que, para esse ponto de corte, a chance de um teste positivo ser verdadeiro é 17,8 vezes maior que a chance de ser falso. Outro fator importante foi o valor do coeficiente Kappa ao comparar o grau de concordância entre o modelo e o padrão-ouro que demonstrou haver grau excelente de concordância. Quando comparamos o diagnóstico clínico da sepse com o padrão-ouro, obtivemos resultados satisfatórios, porém, inferiores ao do modelo testado.

Portanto, os dados analisados oferecem condições satisfatórias para inferirmos que a metodologia aplicada foi adequada e que o modelo apresentado, bem como, o ponto de corte definido tem excelente probabilidade estatística para prever o desenvolvimento de sepse em indivíduos internados em UTI.

Durante a realização da pesquisa algumas limitações importantes se tornaram evidentes e merecem destaque citá-las. Quando analisamos o número de diagnósticos clínicos de sepse do período analisado com o equivalente anteriormente observa-se que ocorreu um decréscimo em torno de $15 \%$, esse fato pode estar associado ao fato dos profissionais da unidade possuírem conhecimento da realização do estudo e terem se tornado mais criteriosos na confirmação da síndrome. Outro fator a ser vislumbrado é o número de indivíduos internados na UTI durante o período estudado. A amostra contou com os registros de 100 indivíduos o que, a priori, parece estatisticamente significativo, contudo, acredita-se que um número maior de informações poderia ter melhorado ainda mais a confiabilidade do modelo testado.

Entretanto, apesar das limitações apresentadas pode-se construir um modelo de decisão probabilístico com excelente poder preditivo para o diagnóstico da sepse sem a necessidade da 
realização da hemocultura, o que além do seu caráter inédito poderá contribuir para a redução da mortalidade ocasionada pela patologia, por apresentar alta confiabilidade associada a baixo custo e resposta rápida.

\section{CONCLUSÃO}

Este estudo objetivou fornecer um modelo de decisão probabilístico para o diagnóstico precoce da sepse, utilizando como principal recurso metodológico para construção do mesmo a regressão logística binária. Identificou-se que a temperatura axilar mínima e máxima, a pressão parcial de $\mathrm{CO}_{2}$, os níveis sanguíneos de lactato, o número de leucócitos totais e de bastonetes são variáveis clínicas que conseguem explicar estatisticamente a ocorrência da síndrome.

A partir dos dados coletados foi possível caracterizar o perfil epidemiológico dos indivíduos internados na UTI do HRP, onde observamos um predomínio do sexo masculino sobre o feminino, maior prevalência de internos acima dos 50 anos, e frequência mais expressiva de patologias clínicas do que cirúrgicas como causas de admissão na unidade, o que se apresenta condizente com o caráter geral do serviço analisado.

Constatou-se a associação das informações clínicas dos pacientes atendidos na UTI no surgimento da sepse, tendo as mesmas se caracterizado como de suma importância para determinação do diagnóstico da patologia através da metodologia utilizada, demonstrando assim sua relevância epidemiológica na determinação da síndrome estudada, como também a necessidade de seu registro de maneira padronizada a fim de prover dados confiáveis para realização de pesquisas como a nossa.

Os resultados também permitiram estabelecer um ponto de corte ideal para a tomada de decisão, o que por sua vez auxiliou na confecção do fluxograma que classifica os pacientes quanto à ocorrência ou não de sepse com base nas variáveis estudadas sem a necessidade de realização de exames onerosos e de resposta tardia como a hemocultura.

Quando comparamos o grau de concordância quanto ao diagnóstico da sepse, entre o modelo apresentado neste estudo e o padrão-ouro relatado pela literatura médica que é a hemocultura utilizando o coeficiente Kappa obtivemos grau de concordância excelente, o que indica boa possibilidade de aplicação prática do modelo avaliado.

Embora a presente pesquisa tenha atingido todos os objetivos inicialmente propostos, devido a amostra analisada ser composta por uma única UTI, recomenda-se a realização de novos estudos com populações de diferentes unidades com o intuito de comprovar os achados aqui mencionados, contribuindo assim para redução da mortalidade decorrente da sepse em todo o mundo. 


\section{REFERÊNCIAS BIBLIOGRÁFICAS}

1 Medeiros LM. Modelo Preditivo Para Diagnóstico Da Sepse Em Unidades De Terapia Intensiva [ Dissertação]. João Pessoa: Universidade Federal da Paraíba, 2012.

2 Dias AT, Matta PO, Nunes WA. Índices de Gravidade em Unidade de Terapia Intensiva Adulto: Avaliação Clínica e Trabalho da Enfermagem. Rev Bras Ter Intensiva 2006; 18(3):123131.

3 Bougard FS, Sue DY. Terapia Intensiva: diagnóstico e tratamento. Porto Alegre: Artmed, 2005.

4 Salles MJ. et al Síndrome da resposta inflamatória sistêmica/sepse - revisão e estudo da terminologia e fisiopatologia. Rev Ass Med Brasil. 1999: 45(1): 86-92.

5 Soane L, et al. Using Quality Improvement Principles to Improve the Care of Patients With Severe Sepsis and Septic Shock. Ochsner J 2013; 13:359-366

6 Yealy DM, Huang DT, Delaney A, Knight M, Randolph AG, Daniels R, Nutbeam T. Recognizing and managing sepsis: what needs to be done? BMC Medicine 2015 13:98 p.2-10.

7 Feijó TR. et al. Gravidade dos Pacientes Admitidos à Unidade de Terapia Intensiva de um Hospital Universitário Brasileiro. Rev Bras Ter Intensiva 2006; 18(1): 18-21.

8 Terzi RG. et al. Índices prognósticos em Medicina Intensiva. Rev Bras Ter Intensiva 2002; 14(1): 6-21

9 IBGE. Pesquisa Nacional por Amostra de Domicílios, 2014

10 Pagano M. Princípios de Bioestatística. São Paulo: CENGAGE, 2013.

11 Medronho R. A. Epidemiologia. São Paulo: Atheneu, 2009.

12 Schisterman, j.L. et al. Linear Regression With an Independent Variable Subject to a Detection Limit. Am J Epidemiol.2006; 163(1): 74-383.

13 Lima ME, Andrade D. Avaliação Prospectiva da Ocorrência de Infecção em Pacientes Críticos de Unidade de Terapia Intensiva. Rev Bras Ter Intensiva 2007; (19)3: 34-46, 
14 Vieira, M. S. Perfil geográfico e clínico de pacientes admitidos na UTI através da Central de Regulação de Internações Hospitalares. Com. Ciências Saúde. 2011; 22(3):201-210

15 Koury GI et al. Fatores de Risco Associados a Mortalidade em Pacientes com Sepse em Unidade de Terapia Intensiva de Hospital Privado de Pernambuco. Rev Bras Terap Intent 2007; 19(1): $23-30$

16 Machado D F. et al. Análise exploratória dos fatores relacionados ao prognóstico em idosos com sepse grave e choque séptico. Rev Bras Ter Intensiva.2009; 21(1):9-17.

17 Carvalho EA, Trotta RA. Avanços no diagnóstico e tratamento da sepse. J Pediatr 2003; 79 (2): S195-S204.

18 Figueiredo RA. et al. Pseudomonas aeruginosas: Frequência de Resistência a Múltiplos Fármacos e Resistência Cruzada entre Microbianos no Recife/PE. Rev Bras Ter Intensiva 2007; 19(4): 253-358.

19 Vieira MA. et al. Avaliação dos Critérios de Definição de Sepse Baseados No "Center For Diseases Control" na Unidade de Terapia Intensiva de Adultos do Hospital de Clínicas da Universidade Federal de Uberlândia. Rev Bras Ter Intensiva. 2009; 22(2): 14- 29.

20 Sousa MA, Medeiros NM, Carneiro JR, Cardoso AM Hemoculturas positivas de pacientes da Unidade de Terapia Intensiva de um hospital escola de Goiânia-GO, entre 2010 e 2013. Estudos; 2014; 41(3): 627-635

21 Zhou J. et al. Epidemiology and Outcome of Severe Sepsis and Septic Shock in Intensive Care Units in Mainland China. PLOS ONE 2014; 9(3): e107181 .

22 Weber RG. et al. Prevalência e Perfil de Resistência de Micro-organismos Isolados de uma UTI de um Hospital da Região Central do Rio Grande do Sul. Rev Prática Méd. 2009; 11(66): 57-63.

23 Makhoul, J. G. et al. Epidemiological, Clinical, and Microbiological Characteristics of Late-Onset Sepsis Among Very Low Birth Weight Infants in Israel: A National Survey. Pediatr 2002; 109(1): 23-29.

24 Batista GK. et al. Avaliação prognóstica individual na UTI: é possível diferenciar insistência terapêutica de obstinação terapêutica? Rev Bras Ter Intensiva 2009; 21(3): 247-254 
25 Guyton AC, Hall JE. Tratado de Fisiologia Médica. Rio de Janeiro: Guanabara Koogan, 2002.

26 Boechat AL, Boechat NO. Sepse: diagnóstico e tratamento. Rev Bras Clin Med.2010; 8(5): 420-427.

27 Noritomi DT. Caracterização Físico-química da acidose metabólica em Paciente com Sepse Grave e Choque Séptico. Faculdade de Medicina da USP. Tese de Doutorado. São Paulo, 2009. $43 p$.

28 Melo WA, Silva J O. Sepse: A importância do Laboratório Clínico no Diagnóstico. Rev Multidisciplinar Saúde 2009; 1(2): 34-50.

29. Kimmoun A. et al. Hemodynamic consequences of severe lactic acidosis in shock states: from bench to bedside. Crit Care. 2015; 9;19:175.

30. Corrar L, Paulo E, Dias Filho J. Análise multivariada para os cursos de administração, ciências contábeis e economia. São Paulo: Atlas, 2007

31. Martinez E . Louzada Neto F, Pereira BB. Analysis of Diagnostic tests using ROC Curves. Cad Saúde Coletiva.2003; 11 (1): 7-31

Artigo apresentado em 19-10-14

Artigo aprovado em 30-01-16 Artigo publicado no sistema em 30-03-16 